\title{
Aspectos sanitários e geração de resíduos na comercialização do pescado na Feira do Ver-o-Peso, Belém, Estado do Pará, Brasil
}

\section{Jônatas Filipe Aires Miranda ${ }^{1}$ e Tiago de Moraes Lenz ${ }^{2}$}

\author{
${ }^{1}$ Universidade Federal do Pará. Curso de Especialização em Gestão Hídrica e \\ Ambiental. Campus Guamá. Rua Augusto Corrêa, 1. Guamá. Belém-PA, Brasil (CEP \\ 66075-110). E-mail: jonatasmiranda00@yahoo.com.br. \\ ${ }^{2}$ Instituto Federal de Alagoas. Campus Penedo. Rodovia Engenheiro Joaquim \\ Gonçalves, S/№. Dom Constantino. Penedo-AL, Brasil (CEP 57200-000).
}

Resumo. As atividades humanas relacionadas à produção e ao consumo promove uma crescente geração de resíduos. Nas grandes cidades, as feiras livres produzem diariamente uma grande quantidade de rejeitos orgânicos que, geralmente, são descartados de forma inadequada, sem receber a devida atenção quanto ao gerenciamento correto dos resíduos produzidos, gerando problemas ambientais e de saúde pública. Dentro do contexto das feiras livres, o setor pesqueiro é um dos que gera diariamente grandes quantidades de resíduos sólidos, devido à evisceração e processamento do pescado. O Estado do Pará destaca-se como segundo maior estado produtor de pescado do país, tendo na sua capital, Belém, o Complexo Mercado do Ver-o-Peso, um dos principais pontos públicos de comercialização e distribuição de pescado do país. 0 objetivo do estudo foi descrever a problemática da produção e descarte de resíduos de pescado no Mercado de Peixe na Feira do Ver-o-Peso, abordando questões de boas práticas de manejo e manipulação do pescado. 0 estudo foi realizado em três etapas que possibilitaram uma abordagem qualitativa e quantitativa da problemática, revisão bibliográfica, observação das estruturas e procedimentos dentro do mercado, bem como entrevistas com os permissionários do Mercado de Peixe da Feira do Ver-o-Peso. Nas observações qualitativas, foi constatado que a estrutura física do mercado encontra-se em boas condições de conservação e o piso é lavado e varrido regularmente, com o recolhimento dos resíduos. Sobre as condições das barracas e do produto, foi observado que há pias e freezers em todos os boxes, porém muitos freezers encontravam-se desligados e as pias não possuíam produtos de limpeza. A principal forma de conservação do pescado é o uso de isopor e gelo e a exposição do produto é feita na bancada da barraca, sem gelo. Os permissionários também não usam uniforme padronizado, sendo que nenhum utilizava luvas. A idade dos permissionários variou de 27 a 80 anos, dos quais 90\% afirmaram que a prefeitura oferece regularmente cursos de manipulação de alimentos. A quantidade de vendas diárias
Recebido

$23 / 07 / 2020$

Aceito

$31 / 03 / 2021$

Disponível on line $04 / 04 / 2021$

Publicado

$30 / 04 / 2021$

Acesso aberto

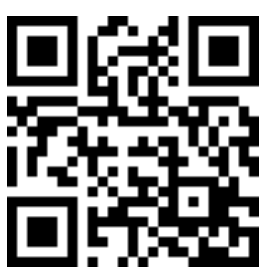

(D) 0000-0002-2495-7881 Jônatas Filipe Aires Miranda

D 0000-0002-7209-8997 Tiago de Moraes Lenz

ISSN 2359-1412/RBGAS-2020-0110/2021/8/18/4/77

Rev. Bras. Gest. Amb. Sustent.

http://revista.ecogestaobrasil.net 
informada pelos entrevistados variaram entre $60 \mathrm{~kg}$ e $200 \mathrm{~kg}$ (média de 137,14 kg \pm 46,89 kg); nossas estimativas são de 4.113 $\mathrm{kg}$ de resíduos gerados diariamente no local, onde atualmente não há coleta seletiva. Com a atual crise dos aterros sanitários em Belém e região metropolitana, a coleta seletiva dos resíduos de pescado com fins de reaproveitamento poderia ser uma boa alternativa para o Mercado de Peixe.

Palavras-chave: Resíduos sólidos; Mercado do Ver-o-Peso; Coleta seletiva.

Abstract. Health aspects and waste generation in the commercialization of fish at the Ver-o-Peso Market, Belém, State of Pará, Brazil. Human activities related to production and consumption have promoted an increasing generation of waste. In large cities, open-air markets produce daily a large amount of organic waste, which is usually disposed of inappropriately and does not receive due attention in relation to the correct management of the waste produced, creating an environmental and public health problem. In the context of open markets, fishing is one of the sectors that generates significant amounts of solid waste daily, due to the evisceration and processing of fish. The state of Pará stands out as the second largest fish producing state in Brazil, having in its capital, Belém, the Ver-o-Peso Market Complex, one of the main public points of commercialization and distribution of fish in the country. The objective of the study was to describe the problem of the production and disposal of fish residues in the Fish Market at the Ver-o-Peso Market, addressing issues of good fish handling. The study was carried out in two stages that enabled a qualitative and quantitative approach to the problem: observation of structures and procedures within the market; and interviews with the salesman of the Fish Market at Ver-o-Peso Market. In qualitative observations, it was found that the physical structure of the market is in good condition and the floor is washed and swept regularly, with the collection of waste. Regarding the conditions of the sales stands and the product, it was observed that there are sinks and freezers in all the boxes, however many freezers were turned off and the sinks did not have cleaning products. The main way to conserve fish is the use of Styrofoam and ice and the product is displayed on the stall bench, without ice. The salesmen also do not wear a standard uniform, none of which used gloves. Their age ranged from 27 to 80 years; $90 \%$ of them stated that the city government regularly offers food handling courses; The daily sales quantities reported by the interviewees ranged from $60 \mathrm{~kg}$ to $200 \mathrm{~kg}$ (average of $137.14 \mathrm{~kg} \pm 46.89 \mathrm{~kg}$ ); our estimates are $4,113 \mathrm{~kg}$ of waste generated daily at the site, where currently there is no waste selective collection. With the current crisis of landfills in Belém and its metropolitan region, the selective collection of fish waste for reuse purposes could be a good alternative for the Fish Market. 
Keywords: Solid waste; Ver-o-Peso Market; Garbage selective collection.

\section{Introdução}

As atividades humanas geram um grande volume de resíduos. Resíduo refere-se a todos os subprodutos e sobras do processamento que são de valor comercial relativamente baixo. Um dos grandes desafios da sociedade moderna é dar um destino adequado aos resíduos e esgoto gerado, tendo em vista que estes afetam diretamente a saúde pública e o meio ambiente (Oliveira et al., 2015).

Dentro das grandes cidades, as feiras livres produzem diariamente uma grande quantidade de rejeitos orgânicos que na maioria dos casos são descartados de forma inadequada, como constatado por Brandão et al. (2014), Alves et al. (2017) e Feitosa et al. (2017). As feiras livres não possuem um plano de gerenciamento dos resíduos produzidos, gerando um problema ambiental e de saúde pública. Um plano de gerenciamento dos resíduos pode mitigar possíveis problemas ambientais decorrentes do descarte inadequado (Cunha, 2013).

0 setor pesqueiro gera diariamente significativas quantidades de resíduos, fruto do processo de beneficiamento do pescado. Este subproduto, rico em proteína, não é aproveitado pela indústria alimentícia, devido principalmente ao restrito conhecimento sobre procedimentos tecnológicos e sanitários para o seu processamento (Pessatti, 2001; Melo et al., 2011). No setor de venda de pescado nas feiras livres, o material residual é constituído geralmente por cabeças, nadadeiras e carcaças, e perfazem de $30 \%$ a $75 \%$ do subproduto gerado (Arruda et al., 2007; Melo et al., 2011).

No Brasil, o Estado do Pará destaca-se como o segundo maior estado produtor de pescado do país (Brasil, 2012). Em sua capital, Belém, o Complexo Mercado do Ver-o-Peso é um dos principais pontos públicos de comercialização e distribuição de pescado do país. Neste contexto, o presente trabalho tem como objetivo descrever a problemática da produção e descarte de resíduos de pescado no Mercado de Peixe na feira do Ver-o-Peso (Belém-PA), abordando questões de boas práticas de manejo e manipulação do pescado.

\section{Material e métodos}

\section{Área de estudo}

A Feira do Ver-o-Peso localiza-se no atual bairro da Campina, às margens da Baía do Guajará. Este tradicional espaço de comércio surgiu em 1627 e passou por adequações e ampliações em 1901, quando foi inaugurado o Mercado de Ferro, também conhecido como Mercado de Peixe do Ver-o-Peso (Fleury e Ferreira, 2011). Ocorreu uma ampla reforma no ano de 2001, onde os azulejos das bancadas das barracas internas do mercado foram substituídos por aço inoxidável.

Atualmente, conta com uma infraestrutura de 60 barracas que comercializam pescado, além de sete estandes que comercializam mariscos (Figura 1 e Figura 2). Todas as barracas e estandes são equipados com energia elétrica e fornecimento de água encanada para suas atividades. As barracas de pescado contêm uma bancada de aço inoxidável, uma pia, um ralo na parte interna e espaço para um freezer e uma balança (Figura 3). Segundo Barthem (2004), desembarcam cerca de 27 toneladas de peixe na Pedra (local de desembarque de barcos pesqueiros em torno do Mercado de Peixe) diariamente. 


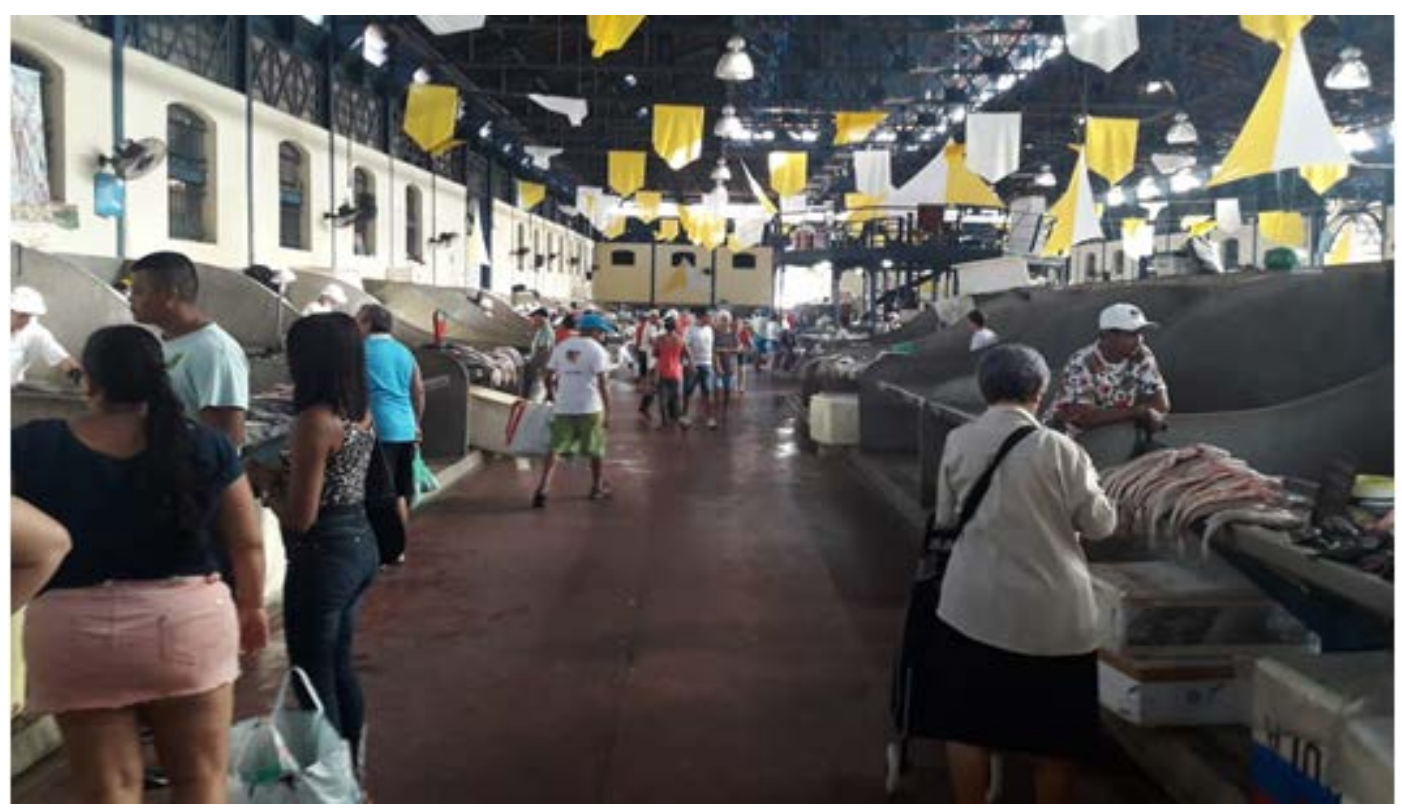

Figura 1. Interior do Mercado de Peixe do Ver o Peso mostrando os estandes de venda de peixe. 2019.

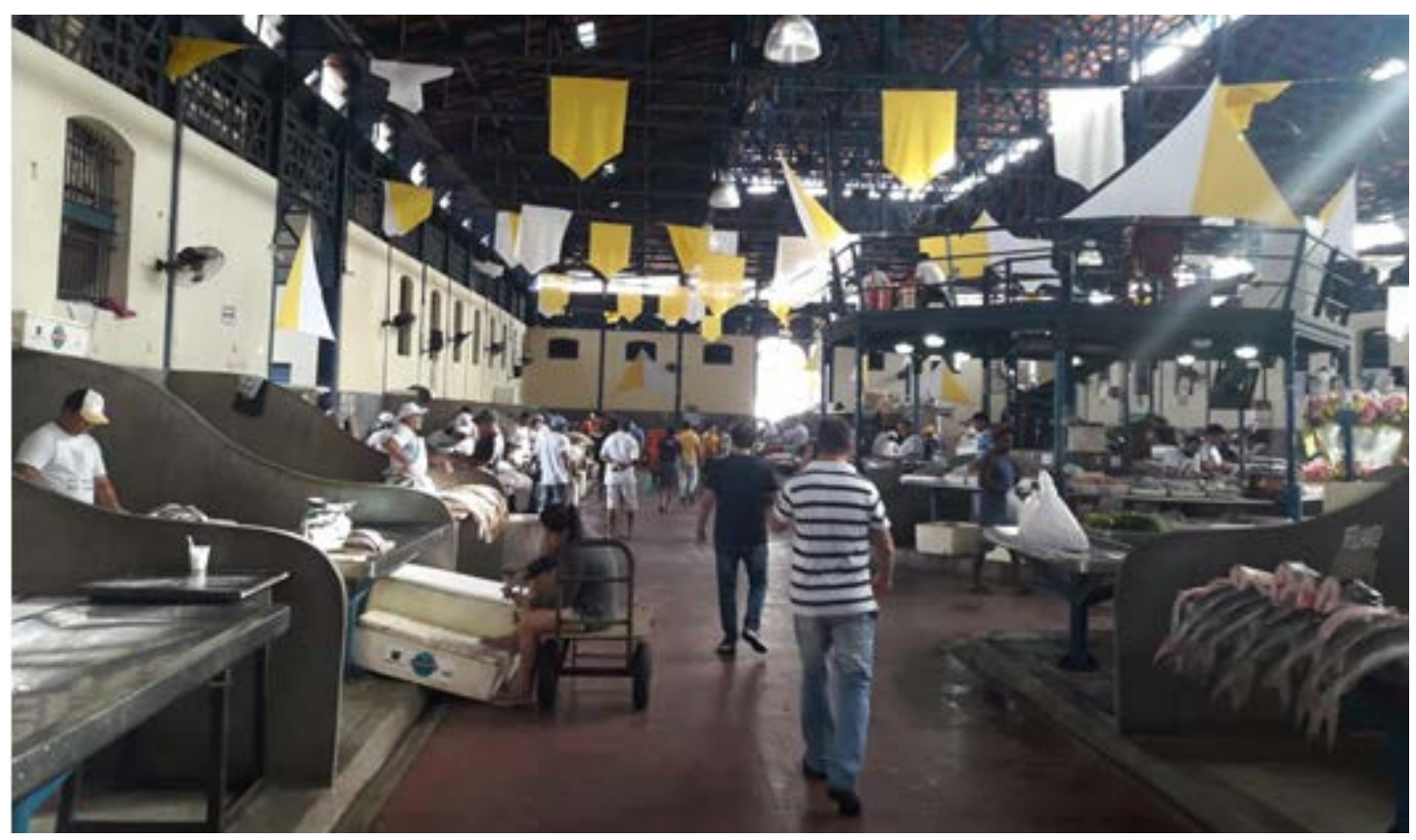

Figura 2. Interior do Mercado de Peixe do Ver o Peso mostrando os estandes de venda de mariscos concentrados no meio do espaço do mercado. 2019. 


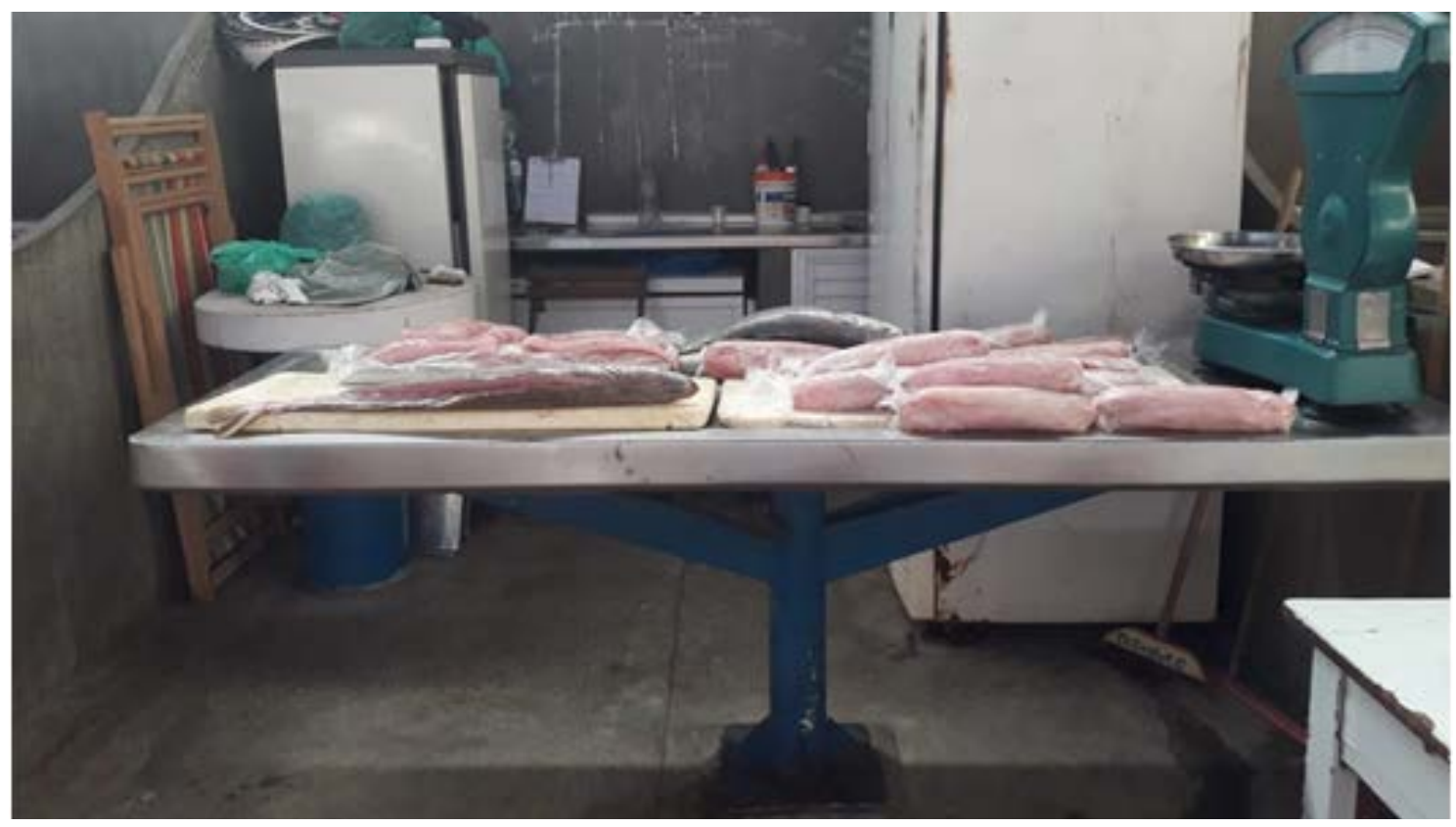

Figura 3. Barraca de venda de peixe, contendo uma bancada de aço inoxidável, uma balança analógica, alguns refrigeradores e uma pia com armário para armazenar produtos de higiene e outras ferramentas do permissionário. 2019.

\section{Metodologia}

O estudo consistiu na observação das estruturas e procedimentos dentro do mercado e na realização de entrevistas com os feirantes do Mercado do Peixe da Feira do Ver-o-Peso em Belém do Pará.

No mês de janeiro de 2019, foi realizada a observação geral sobre a condição sanitária da feira, levando em conta a condição aparente dos boxes, a estrutura geral do mercado, a presença de equipamentos para o armazenamento/conservação do pescado, bem como o material de manipulação do produto usado pelos permissionários. Posteriormente, foram aplicadas as entrevistas aos permissionários através de um questionário predefinido. Todos os entrevistados ficaram cientes do teor do estudo, seus objetivos e a instituição onde os resultados seriam apresentados. Todos os participantes assinaram voluntariamente um termo de consentimento livre e esclarecido, onde constavam as informações do estudo e seus responsáveis. Do total de 60 barracas que estavam funcionando, as entrevistas foram feitas com 10 feirantes, $(16,66 \%)$, oito comercializavam peixes e dois, mariscos.

As informações quantitativas foram estimadas através das respostas dos entrevistados, através da relação quantidade de pescado vendida diariamente, pela proporção de resíduos gerados (\%) a cada kg de peixe vendido, chegando a um valor diário.

Para a estimativa da quantidade de peixe vendida diariamente, foi calculada uma média de todos os valores informados pelos entrevistados. Caso tenha sido informado um intervalo, utilizou-se o valor central deste (e.g., quantidade informada: 100-150 kg; valor usado na estimativa: $125 \mathrm{~kg}$ ). Após calcular a média de quantidade vendida informada pelos entrevistados, este valor foi extrapolado para o número total de barracas do mercado. 
A estimativa da quantidade de resíduos gerada diariamente foi calculada levando em conta o percentual de resíduos em cada quilograma de peixe comercializado. Vale ressaltar que o percentual de resíduos gerados por peixe é variável de acordo com o tamanho, espécie e tipo de beneficiamento. Valores entre 30\% e 70\% de resíduos gerados são relatados no trabalho de Souza e Inhamuns (2011) para o pescado de 'corpo limpo' (i.e., sem cabeça, vísceras, nadadeiras e escamas) e filetado sem pele, respectivamente. Também podemos encontrar valores médios em torno de $50 \%$ de resíduos em relação ao peso total do pescado (Pessatti, 2001; Arruda et al., 2007; Leite et al., 2016), o que se aproximou da estimativa feita pelos entrevistados. Sendo assim, o valor utilizado neste trabalho para estimar a geração de resíduos foi de $50 \%$ do peso médio comercializado.

0 questionário aplicado foi do tipo semiestruturado através de entrevista aberta e informal, possibilitando adicionar informações relatadas em cada item questionado. 0 resultado final do estudo foi uma junção das respostas obtidas nas entrevistas com a observação das condições sanitárias do mercado.

\section{Resultados}

\section{Condições gerais do Mercado de Peixe}

As observações mostraram que a estrutura física do mercado se encontra em boas condições. $O$ recinto apresenta paredes e pisos revestidos de material impermeável de fácil higienização. A lavagem do piso e a retirada dos resíduos em um contêiner ocorrem diariamente mais de uma vez, segundo os entrevistados (Figura 4). Não se observou animais domésticos e tampouco pássaros dentro do espaço do mercado durante a visita.

Próximo a uma das entradas do mercado encontrava-se um cartaz feito pelos próprios permissionários em que constava um aviso sobre uma contribuição mensal de cada trabalhador para custear a manutenção de limpeza do mercado. A prefeitura delegou aos permissionários a responsabilidade da manutenção e limpeza dentro do espaço físico do mercado. Esta função é desempenhada por uma comissão rotativa composta por quatro membros, a qual fica responsável pela arrecadação mensal para custear os serviços de varrição, lavagem, coleta de resíduos, eventuais manutenções físicas de pequeno porte na infraestrutura do mercado e barracas, e até serviço de vigilância.

Há resistência por parte dos feirantes em se envolver com esta função. Dentre os entrevistados, dois faziam parte da comissão (um deles era o atual presidente) e ambos afirmaram que a função traz mais problemas do que benefícios e que gostariam que o poder público fosse mais presente dentro do mercado.

Quanto às condições das barracas foi observado a presença de pias e freezers em todos os boxes, porém muitos freezers encontravam-se desligados e as pias não possuíam produtos de limpeza. A exposição do produto para a venda é feita na bancada da barraca, sem o uso de gelo (Figura 3).

Nem todos os permissionários usavam aventais e toucas, e não foi encontrado nenhum que estivesse usando luvas para manipular o pescado. As facas, em geral, encontravam-se aparentemente em boas condições de uso, porém, ao considerarmos que em nenhuma barraca foram encontrados detergentes para a lavagem das ferramentas, é possível que as condições higiênicas das facas estivessem comprometidas. Os removedores de escamas, em sua maioria, fabricados artesanalmente, encontravam-se bastante oxidados, comprometendo a higiene do beneficiamento do pescado. 


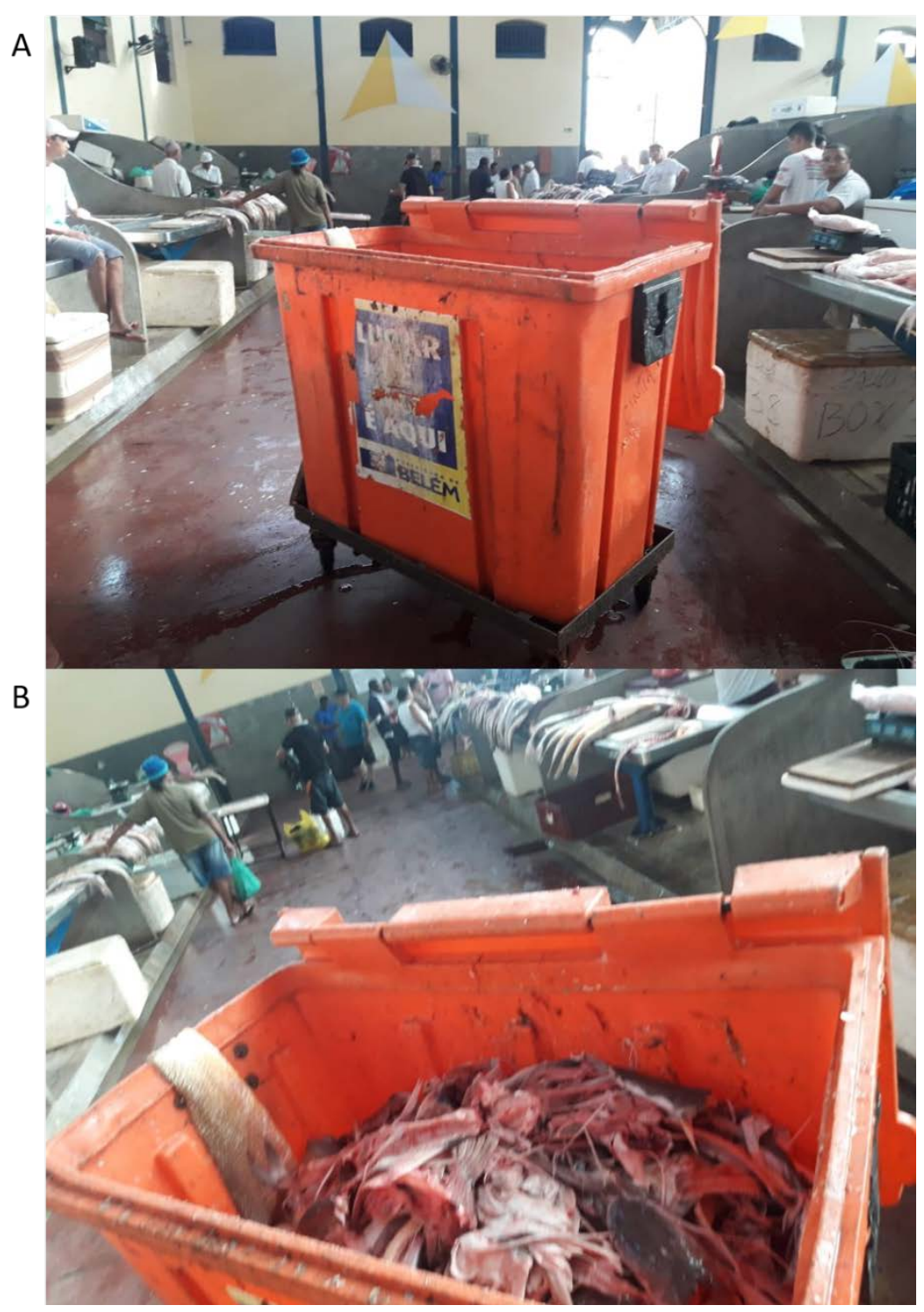

Figura 4. A- Contêiner usado na retirada de resíduos de pescado do Mercado de Peixe. BQuantidade de resíduos retirados em uma das limpezas regulares que ocorrem várias vezes ao dia, segundo os permissionários. 2019.

\section{Entrevista}

Os dados socioeconômicos são mostrados na Tabela 1. Sobre a capacitação, $90 \%$ dos entrevistados afirmaram que a prefeitura de Belém oferece regularmente cursos de manipulação de alimentos e exige que os mesmos possuam, além do referido curso, um certificado de boas condições de saúde para poder trabalhar no Mercado de Peixe. 
Tabela 1. Dados socioeconômicos dos permissionários entrevistados. 0 item "Renda" está baseado no valor do salário mínimo vigente, em janeiro/2019 (R\$998,00).

\begin{tabular}{crccc}
\hline Barraca & Idade & Escolaridade & Experiência & Renda (No de salários mínimos) \\
\hline A & 51 & Fundamental (i) & 35 & $1-2$ \\
\hline B & 33 & Fundamental (c) & 15 & $>2$ \\
\hline C & 80 & Fundamental (c) & 65 & $>2$ \\
\hline D & 36 & Fundamental (c) & 20 & $>2$ \\
\hline E & 27 & Médio (i) & 12 & $1-2$ \\
\hline F & 53 & Médio (c) & 40 & $1-2$ \\
\hline G & 32 & Médio (c) & 10 & $1-2$ \\
\hline H & 64 & Médio (c) & 51 & $1-2$ \\
\hline I & 48 & Fundamental (i) & 35 & $>2$ \\
\hline J & 45 & Fundamental (i) & 5 & $<1$ \\
\hline
\end{tabular}

As espécies comercializadas pelos permissionários estão na Tabela 2.

A origem do pescado é predominantemente das embarcações que atracam diariamente em torno do Mercado Ver-o-Peso. Os permissionários também, eventualmente, recebem o pescado de caminhões, vindos geralmente do nordeste do estado com outras espécies, como a tainha Mugil spp. e pargo Lutjanus spp.

Sobre a conservação e armazenamento do pescado, as respostas constam na Figura 5.

Tabela 2. Espécies de peixes e mariscos comercializados regularmente no Mercado de Peixe, ordenadas pela procura e importância nas vendas, segundo informações dos permissionários.

\begin{tabular}{ll}
\hline \multicolumn{1}{c}{ PEIXES } & \\
\hline Espécie & Nome popular \\
\hline Cynoscion acoupa (Lacepède, 1801) & Pescada amarela \\
\hline Brachyplatystoma flavicans (Castelnau, 1855) & Dourada \\
\hline Brachyplatystoma filamentosum (Lichtenstein, 1819) & Filhote \\
\hline Cynoscion leiarchus (Cuvier, 1830) & Pescada branca \\
\hline Cynoscion microlepidotus (Cuvier, 1830) & Pescada gó \\
\hline Arius luniscutis (Valenciennes, 1840) & Gurijuba \\
\hline Brachyptalystoma vaillantii (Valenciennes, 1840) & Piramutaba \\
\hline Arapaima gigas (Cuvier, 1829) & Pirarucu \\
\hline \multicolumn{1}{c}{ MARISCOS } & \\
\hline Espécie & Nome popular \\
\hline Farfantepenaeus subtilis (Peréz Farfante, 1967) & Camarão rosa \\
\hline Machobrachium amazonicum (Heller, 1862) & Camarão da Amazônia \\
\hline Macrobrachium rosenbergii (De Man, 1879) & Camarão da Malásia \\
\hline Ucides cordatus (Linnaeus, 1763) & Caranguejo uçá \\
\hline
\end{tabular}




\section{CONSERVAÇÃO E ARMAZENAMENTO DO PESCADO}

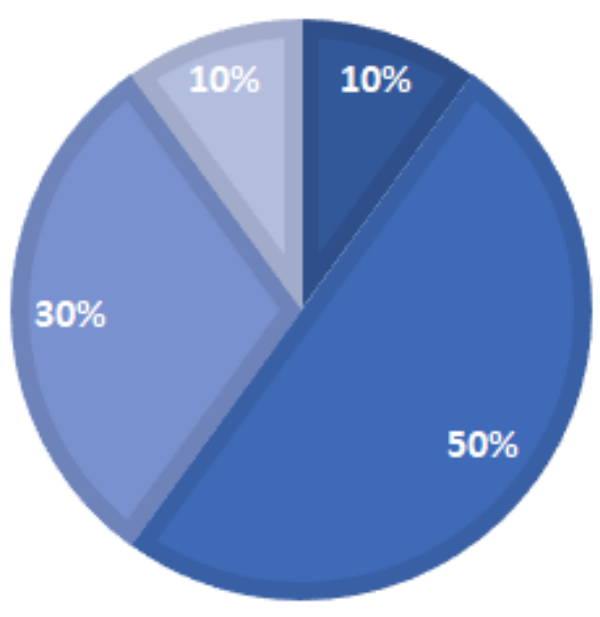

Freezer $\mathbf{m}$ Freezer e isopor $\mathbf{m}$ Isopor $\mathbf{m}$ Outro (freezer desligado)

Figura 5. Forma de conservação e armazenamento do pescado para a venda. É importante destacar que todas as barracas possuíam freezer e energia elétrica.

Em relação aos resíduos, os vendedores de mariscos afirmaram que não geram uma quantidade grande de resíduos, pois seu produto é vendido sem passar pelo beneficiamento, portanto as informações utilizadas nesta parte do estudo foram apenas dos vendedores de peixes. Um dos oito vendedores de peixe disse não saber informar a quantidade de resíduos gerada no dia, portanto a média foi calculada com base nos dados dos sete que informaram.

Tabela 3. Quantitativo de vendas (kg) e geração de resíduos (kg e \%) por dia, acompanhados das respectivas médias. As oito barracas estão identificadas por letras e a quantidade de resíduos da barraca G não foi informada, portanto seus dados não foram incluídos no cálculo das médias.

\begin{tabular}{cccc}
\hline Barraca & Venda (kg/dia) & Resíduos (kg/dia) & \% resíduos \\
\hline A & 175 & 87,5 & 50 \\
\hline B & 150 & 60 & 40 \\
\hline C & 150 & 65 & 43 \\
\hline D & 100 & 40 & 40 \\
\hline E & 200 & 100 & 50 \\
\hline F & 125 & 62,5 & 50 \\
\hline G & 90 & Não informou & - \\
\hline H & 60 & 27,5 & 46 \\
\hline Média (desvio) & $137,14( \pm 46,89)$ & $63,21( \pm 25,07)$ & $46( \pm 0,04)$ \\
\hline
\end{tabular}


As quantidades de vendas diárias informadas pelos entrevistados variaram entre $60 \mathrm{~kg}$ e $200 \mathrm{~kg}$ (média de 137,14 kg $\pm 46,89 \mathrm{~kg}$ ). 0 valor extrapolado para as 60 barracas, resultou em uma média diária de $8.226 \mathrm{~kg}$ de peixe comercializados diariamente dentro do Mercado. Aplicando a taxa de $50 \%$ de geração de resíduos em relação à quantidade vendida, o total de resíduos gerados diariamente dentro do Mercado é de $4.113 \mathrm{~kg}$.

A Figura 6 mostra o destino dado aos resíduos produzidos no Mercado de Peixe. Apesar de $40 \%$ afirmarem que há coleta seletiva, esta informação parece estar desatualizada, pois nenhum entrevistado soube informar o nome da empresa que realiza este serviço.

Em relação ao destino dos resíduos do Mercado, todos os entrevistados afirmaram que a coleta seletiva é a melhor opção, já que podem ser reaproveitados. A nota média para as condições sanitárias do mercado foi 8 (variando de 7 a 10), mostrando que, no geral, os trabalhadores estão satisfeitos com as condições higiênicas. Em relação à atuação do poder público na manutenção do mercado, todos os entrevistados foram unanimes que, se não fosse por eles, as condições do mercado estariam bem piores. A última questão foi sobre o que poderia melhorar no cuidado do poder público em relação ao mercado (Figura 7).

\section{DESTINO DOS RESÍDUOS}

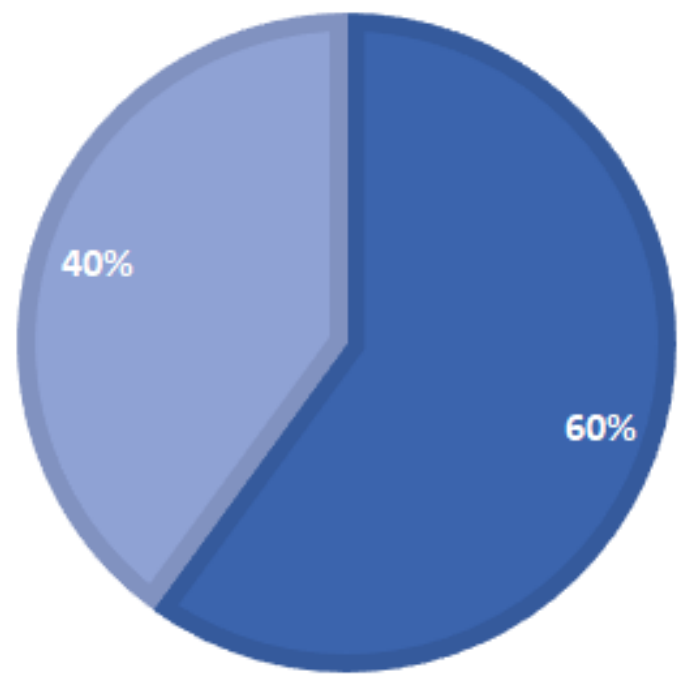

- Coleta regular/lixo comum Coleta seletiva

Figura 6. destino dos resíduos de pescado produzidos dentro do Mercado de Peixe, segundo informações dos permissionários. 


\section{O QUE PODE MELHORAR?}

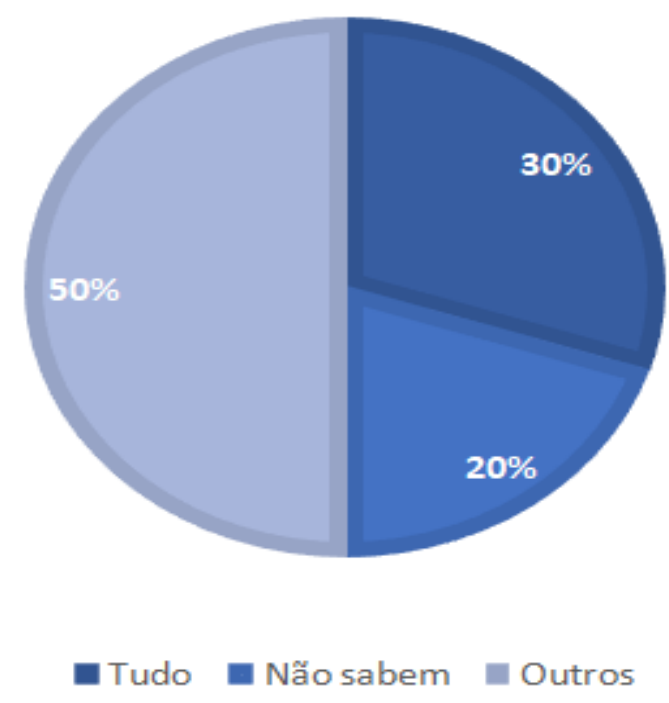

Figura 7. Opinião dos permissionários sobre pontos que devem ser melhorados a respeito do cuidado do poder público com o Mercado de Peixe. *Outros: a fiscalização da qualidade do pescado vendido; a questão da segurança e higiene do mercado; e aumentar a responsabilidade da prefeitura.

Ao fim da entrevista foi deixado um espaço para comentários adicionais dos entrevistados, no qual oito entrevistados reclamaram do comércio informal de peixe ao redor do mercado, o qual prejudica suas vendas dentro do mercado. Outra reclamação e que os vendedores externos descartam os resíduos do beneficiamento nas calçadas, atraindo insetos e outros animais, que eventualmente adentram o espaço do mercado e comprometem as condições sanitárias deste.

\section{Discussão}

O ambiente de feiras livres apresenta características favoráveis para contaminação de micro-organismos e sua proliferação, devido, entre outros fatores, a produtos comercializados sem a higienização adequada e manipuladores utilizando adornos e sem o uso de uniformes padronizados (Xavier et al., 2009). Foi observada uma boa higienização aparente dos estabelecimentos, porém nenhum dos permissionários usava luvas, tampouco havia detergente para higienização das mãos e utensílios. Outro agravante observado foi que os mesmos trabalhadores que manipulam os alimentos realizam tarefas diversas, tais como varrição, retirada do lixo, recepção do dinheiro e outros, podendo isso causar contaminação do alimento comercializado (Sousa et al., 2011; Dias et al., 2012).

Segundo Capistrano et al. (2004), em feiras-livres é comum que barracas convivam lado a lado devido à limitação de espaço, gerando um risco de que as más condições sanitárias de uma barraca sejam propagadas para as outras. No Mercado de Peixe é notável a homogeneidade das barracas e um espaço relativamente amplo, porém é possível que as más práticas de higiene de alguns permissionários ofereçam risco às condições sanitárias de barracas próximas. A contaminação por microrganismos presentes 
em alimentos crus ocorre preferencialmente pelas mãos dos manipuladores e dos utensílios de cozinha, que constituem veículos comuns para a transmissão de agentes de toxinfecções alimentares (Cunha, 2013).

Em relação à conservação, a ausência de gelo nas bancadas para a exposição do pescado contribui para a diminuição da qualidade do produto. A temperatura é o principal fator que determina a velocidade de deterioração do pescado (Gaspar et al., 1997; Bartolomeu et al., 2011; e Mol e Tosun, 2011), que é um alimento muito rico e saudável, mas se não for bem conservado, pode se tornar vilão da alimentação, causando sérios problemas de intoxicação e infecção a quem o consumir (Vieira, 2004; Alves et al., 2017).

0 acondicionamento do pescado pelos permissionários ocorre principalmente com o uso de isopores e, em alguns casos, freezers. A Portaria MAPA no 185/1997 (Brasil, 1997), que trata da qualidade para a comercialização do peixe fresco, determina que o acondicionamento do peixe para a venda deve dar-se com a conservação feita por uma camada de gelo triturado, assegurando uma temperatura próxima à fusão do gelo. Apesar das temperaturas não terem sido medidas, o estudo de Alves et al. (2017) relata temperaturas médias do pescado exposto na bancada (nas mesmas condições que as encontradas neste estudo) de $22{ }^{\circ} \mathrm{C}$. Outros autores relataram essa mesma problemática em outras feiras livres, como Cunha (2013), em Manaus-AM, Brandão et al. (2014), em Santarém-PA, e Silva et al. (2014), no Distrito de Icoaraci (Belém-PA).

Os dados socioeconômicos mostram que, apesar das diferenças de idade entre os trabalhadores, quase todos possuíam pelo menos 10 anos de experiência na atividade. A prefeitura de Belém faz algumas exigências em relação à qualificação deles e oferta cursos de capacitação periodicamente. 0 treinamento e conscientização dos feirantes são de grande importância para garantir a segurança alimentar de seu produto evitando problemas de saúde pública (Góes, 2001) e, neste quesito, percebe-se um acerto do poder público.

O pescado comercializado depende de vários fatores, desde seu preço cobrado pelos fornecedores até sua qualidade, sendo que algumas barracas possuíam grande variedade de espécies enquanto outras vendiam apenas uma. 0 período de defeso de algumas espécies também pode influenciar no pescado disponível para venda. Assim como o encontrado por Alves et al. (2007), os permissionários não souberam informar com precisão de onde vinha o produto que eles comercializavam, sendo que a maioria chega em barcos.

Tratando-se da geração de resíduos, segundo as estimativas feitas, a quantidade diária chegou a $4.113 \mathrm{~kg}$. A maioria dos clientes que compra peixes solicita que o permissionário faça o beneficiamento do pescado. Neste processo ocorre a geração de resíduos compostos basicamente por cabeças, vísceras, nadadeiras, peles, escamas e espinhos (SEBRAE, 2010) e podem representar mais de 50\% da matéria-prima utilizada, variando conforme as espécies e o processamento (Feltes et al., 2010). Autores como Valente et al. (2014), atribuem uma porcentagem ainda maior quanto a geração de resíduos de pesca, de $65 \%$ do peso do pescado.

De acordo com as normas brasileiras regentes, os resíduos de pescado podem ser classificados de acordo com a ABNT NBR 10.004:2004 (ABNT, 2004) e a Lei no 12.305/2010, da Política Nacional de Resíduos Sólidos (Brasil, 2010), como Classe II (não inertes), que possuem propriedades como biodegradabilidade, combustibilidade, ou solubilidade em água, como resíduos de pescado não contaminados. Os resíduos de Classe II da indústria pesqueira e da aquicultura são aqueles com maior potencial para a reciclagem (SEBRAE, 2010).

Os resíduos gerados no Mercado de Peixe poderiam ser aproveitados caso houvesse coleta seletiva e alguma empresa com condições de fazer esse serviço. Segundo Arruda (2004), os resíduos do beneficiamento do pescado podem ser direcionados para quatro categorias de aproveitamento: alimento para consumo humano, ração animal, 
fertilizantes e produtos químicos. A maioria do resíduo aproveitado é direcionada para a produção de farinha de peixe, um tipo de ração animal (López-Mosquera et al, 2011).

0 aproveitamento de resíduos da cadeia produtiva do pescado pode constituir um eficaz aliado na diminuição do desperdício e, consequentemente, mitigação da poluição ambiental, pois com a criação de alternativas tecnológicas com valor agregado que permitam o gerenciamento dos resíduos sólidos de pescado, virá a segurança alimentar, o combate à fome e o desenvolvimento sustentado (Espindola Filho, 1997).

Além da produção da farinha de peixe, que traz um fraco retorno financeiro, Arruda et al. (2007) recomendam como alternativa, a silagem dos resíduos de peixe, que consiste em um processo de baixo custo em que um subproduto liquefeito é gerado. Valente et al. (2016), por sua vez, recomendam a produção de fertilizante orgânico a partir da compostagem de resíduos de pescado e arroz, afirmando que a compostagem é um meio eficiente de decomposição dos resíduos do pescado. Ainda há outras alternativas de reaproveitamento dos resíduos, como a produção de medicamentos e biocombustíveis (Ghaly et al., 2013).

Ficou evidente que a situação atual do Mercado de Peixe em relação à geração e aproveitamento de resíduos precisa ser discutida, pois não conta com nenhuma estratégia para o reaproveitamento, agravando a já existente crise dos aterros sanitários vivida pela Grande Belém, devido à desativação do aterro sanitário de Marituba previsto para junho de 2021 (G1 Pará, 2019; Portal Belém, 2020). Este é um nicho disponível para ser explorado, tanto pela iniciativa privada quanto pelo poder público. Estas ações devem ser alvo de políticas públicas, as quais contribuiriam para a melhoria da condição sanitária do local, diminuiria o volume de lixo produzido e geraria empregos para o seu beneficiamento.

\section{Conclusões}

O espaço possui boas condições aparentes de higiene, (retirada de resíduos dos corredores, varrição e lavagem do piso). A maioria dos permissionários possui mais de 10 anos de experiência profissional e, apesar do baixo grau de escolaridade ser predominante entre eles, todos realizam regularmente cursos de capacitação oferecidos pela prefeitura de Belém. No entanto, foram identificadas algumas práticas não recomendáveis na manipulação de alimentos como manuseio do produto sem a utilização de luvas e, em alguns casos, sem aventais e toucas também, ausência de produtos de higiene nas barracas para lavar as mãos e ferramentas, e exposição do pescado nas bancadas sem gelo para conservá-lo.

Estima-se que cerca de $4.113 \mathrm{~kg}$ de resíduos são gerados diariamente no Mercado de Peixe. Não existe coleta seletiva atualmente no Mercado, sendo todo esse resíduo descartado nos contêineres de lixo comum alocados no exterior do mercado e coletado pelo serviço de coleta regular de lixo da prefeitura.

Algumas alternativas ao descarte comum do resíduo de pescado devem ser consideradas, já que o descarte desse material em depósitos comuns de lixo aumenta a degradação ambiental dessas áreas. Os resíduos de pescado possuem um bom potencial para reciclagem, podendo gerar diversos produtos, como farinha de peixe, fertilizantes orgânicos e silagem.

\section{Conflito de interesses}

Os autores declaram não haver conflito de interesses. 


\section{Referências}

ABNT - Associação Brasileira de Normas Técnicas. ABNT NBR 10004: Resíduos sólidos Classificação. Rio de Janeiro: ABNT, 2004.

Alves, E. M. P.; Silva, A.; Sousa, C. L.; Figueiredo, H. M.; Neves, E. A. Aspectos higiênicossanitários das instalações do Mercado do Ver-o-Peso e avaliação da temperatura dos peixes comercializados. Revista Eletrônica da FAINOR, v. 10, n. 2, p.25-43, 2017.

Arruda, L. F.; Borghesi, R.; Oetterer, M. Use of fish waste as silage: A review. Brazilian Archives of Biology and Technology, v. 50, n. 5, p. 879-886, 2007.

Barthem, R. B. O desembarque na região de Belém e a pesca na foz amazônica. In: Rufino, M. L. (Ed.). A pesca e os recursos pesqueiros na Amazônia brasileira. Manaus: Pro-Várzea, 2004. p. 138-167.

Bartolomeu, D. A. F. S.; Dallabona, D. R.; Macedo, R. E. F.; Kirschnik, P. G. Contaminação microbiológica durante as etapas de processamento de filé de tilápia (Oreochromis niloticus). Archives of Veterinary Science, v. 16, n. 1, p. 21-30, 2011. https://doi.org/10.5380/avs.v16i1.22788

Brandão, B. P.; Vinente Neto, B. F.; Pontes, D. F.; Carvalho, J. A. S.; Leal, J. F. S.; Quaresma, T. C. Agravantes ambientais que influenciam na carne e no pescado do Mercado Municipal de Santarém-PA. Em Foco, v. 1, n. 21, p. 21-27, 2014.

Brasil. Boletim Estatístico da Pesca e Aquicultura. Brasília: CEPSUL, 2012. Disponível em: <https://www.icmbio.gov.br/cepsul/images/stories/biblioteca/download/estatistica/ est_2011_bol_bra.pdf $>$. Acesso em: 28 jul. 2019.

Brasil. Lei no 12.305, de 2 de agosto de 2010. Institui a Política Nacional de Resíduos Sólidos; altera a Lei no 9.605, de 12 de fevereiro de 1998; e dá outras providências. Disponível em: <http://www.planalto.gov.br/ccivil_03/_ato2007-2010/2010/lei/ 112305.htm>. Acesso em: 16 jul. 2019.

Brasil. Portaria MAPA no 185, de 19 de maio de 1997. Regulamento Técnico de Identidade e Qualidade de Peixe Fresco (inteiro e eviscerado). Disponível em: <https://www.defesa.agricultura.sp.gov.br/legislacoes/portaria-mapa-185-de-13-051997,670.html>. Acesso em: 15 jul. 2019.

Brasil. Resolução RDC 216, de 15 de setembro de 2004. Regulamento técnico de boas práticas para serviços de alimentação. Disponível em: <http://portal.anvisa.gov.br/ documents/33916/388704/RESOLU\%C3\%87\%C3\%830-RDC+N+216+DE+15+DE+ SETEMBRO+DE+2004.pdf/23701496-925d-4d4d-99aa-9d479b316c4b>. Acesso em: 16 jul. 2019.

Capistrano, D. L.; Germano, P. M. L.; Germano, M. I. S. Feiras-livres do Município de São Paulo sob ponto de vista legislativo e sanitário. Revista Higiene Alimentar, v. 18, n. 116/117, p. 37-41, 2004.

Cunha, E. A. B. Aspectos sanitários e de gestão ambiental do setor de pescado em uma feira livre no Município de Manaus. Belém: Universidade Federal do Pará, 2013. (Dissertação de mestrado).

Dias, M. A. C.; Sant'Ana, A. S.; Cruz, A. G.; Faria, J. A. F.; Oliveira, C. A. F.; Bona, E. On the implementation of good manufacturing practices in a small processing unity of mozzarella cheese in Brazil. Food Control, v. 24, n. 1/2, p. 199-205, 2012. https://doi.org/10.1016/ j.foodcont.2011.09.028 
Espíndola Filho, A. Aproveitamento de resíduos sólidos de pescado como fertilizante marinho. São Paulo: Universidade Mackenzie, 1997. (Dissertação de mestrado).

Feitosa, G. P.; Silva, G. V. G. M.; Lima, M. J. F.; Sousa, G. A. M.; Silva, I. C. M.; Sousa, K. N. S. Boas práticas na manipulação de pescado como capacitação para manipuladores de pescado de Santarém, Pará, Brasil. Revista Brasileira de Engenharia de Pesca, v. 10, n. 1, p. 16-26, 2017.

Feltes, M. M. C.; Correia, J. F. G.; Beirão, L. H.; Block, J. M.; Ninow, J. L.; Spiller, V. R. Alternativas para a agregação de valor aos resíduos da industrialização de peixe. Revista Brasileira de Engenharia Agrícola e Ambiental, v. 14, n. 6, p. 669-677, 2010.

Fleury, J. N.; Ferreira, A. A. Ver-o-Peso da cidade: o mercado, a carne e a cidade no final do século XIX. Revista Estudos Amazônicos, v. 6, n. 1, p. 100-116, 2011.

G1 Pará. Belém ainda discute o que fazer com o lixo de 2,5 milhões de paraenses. Belém, 2019. Disponível em: <https://g1.globo.com/pa/para/noticia/2019/06/05/belem-aindadiscute-o-que-fazer-com-o-lixo-de-25-milhoes-de-paraenses.ghtml>. Acesso em: 19 jul. 2019.

Gaspar, J.; Vieira, R.; Tapia, M. Aspectos sanitários do pescado de origem de água doce e marinha, comercializado na Feira de Gentilândia, Fortaleza, Ceará. Revista Ciência e Tecnologia de Alimentos, v. 11, p. 20-287, 1997.

Ghaly, A. E.; Ramakrishnan, V. V.; Brooks, M. S.; Budge, S. M.; Dave, D. Fish processing wastes as a potential source of proteins, amino acids and oils: A critical review. Microbial \& Biochemical Technology, v. 5, n. 4, p. 107-129, 2013. https://doi.org/10.4172/19485948.1000110

Góes, J. A. W. Capacitação dos manipuladores de alimentos e a qualidade da alimentação servida. Revista de Higiene Alimentar, v. 15, n. 81, p. 20-22, 2001.

Leite, S. B. P.; Sucasas, L. F. A.; Oetterer, M. Resíduos da comercialização de pescado marinho: volume de descarte e aspectos microbiológicos. Revista Brasileira de Tecnologia Agroindustrial, v. 10, n. 1, p. 2112-2125, 2016.

López-Mosquera, M. E.; Fernández-Lema, E.; Vilhares, R.; Corral, R.; Alonso, B.; Blanco, C. Composting fish waste and seaweed to produce a fertilizer for use in organic agriculture. Procedia Environmental Sciences, v. 9, p. 113-117, 2011. https://doi.org/10.1016/ j.proenv.2011.11.018

Melo, F. O.; Alves, M. M.; Guimarães, M. D. F.; Holanda, F. C. A. F. Aproveitamento do resíduo a partir do beneficiamento de pescado de uma indústria pesqueira no norte do Brasil. Arquivos de Ciências do Mar, v. 44, n. 3, p. 5-11, 2011.

Mol, S.; Tosum, S. Y. The quality of fish in retail markets in Istanbul, Turkey. Journal of Fisheries Sciences, v. 5, n. 1, p. 16-25, 2011. https://doi.org/10.3153/jfscom.2011002

Oliveira, E. G.; Oliveira, A. G.; Albuquerque, M. V. C. Avaliação da gestão dos resíduos gerados em uma feira livre na Cidade de Campina Grande-PB. Anais do Congresso Brasileiro de Gestão Ambiental e Sustentabilidade - Congestas 2015, v. 3, p. 380-391, 2015. Disponível em: <http://eventos.ecogestaobrasil.net/congestas2015/trabalhos/ pdf/congestas2015-et-03-028.pdf>. Acesso em: 16 jul. 2019.

Pessatti, M. L. Aproveitamento dos subprodutos do pescado: meta 11. Santa Catarina: Universidade do Vale do Itajaí, 2001. (Relatório final de ações prioritárias ao desenvolvimento da pesca e aquicultura no sul do Brasil, convênio Ministério da Agricultura, Pecuária e Abastecimento - MAPA).

Rev. Bras. Gest. Amb. Sustent., 2021, vol. 8, n. 18, p. p. 77-92. 
Portal Belém. Destinação do lixo da Região Metropolitana é tema de reunião. Belém, 2020. Disponível em: <https://belem.com.br/noticia/1797/destinacao-do-lixo-da-regiaometropolitana-e-tema-de-reuniao>. Acesso em: 26 fev. 2020.

SEBRAE - Serviço Brasileiro de Apoio às Micro e Pequenas Empresas. Diagnóstico dos resíduos da pesca e aquicultura do Espírito Santo. Vitória: SEBRAE, 2010.

Silva, W. B.; Nogueira, J. G.; Pena A. H. Avaliação das condições higiênico-sanitárias na comercialização do pescado no Mercado Municipal de Icoaraci/Pará-Brasil. Observatorio de la Economía Latinoamericana, n. 200, 2014. Disponível em: <http://www.eumed.net/cursecon/ecolat/br/14/higiene.hmtl>. Acesso em: 13 jan. 2019.

Sousa, C. L.; Freitas, J. A.; Lourenço, L. F. H.; Araújo, E. A. F.; Souza, J. N. S. Avaliação da qualidade microbiológica no processamento de pescados. Revista do Instituto Adolfo Lutz, v. 70, n. 2, p. 150-156, 2011.

Souza, A. F. L.; Inhamuns, A. J. Análise de rendimento cárneo das principais espécies de peixes comercializadas no Estado do Amazonas, Brasil. Acta Amazonica, v. 41, n. 2, p. 289-296, 2011.

Valente, B. S.; Xavier, E. G.; Pereira, H. S.; Pilotto, M. V. T. Compostagem na gestão de resíduos de pescado de água doce. Boletim do Instituto de Pesca, v. 40, n. 1, p. 95-103, 2014. Disponível em: <ftp://ftp.sp.gov.br/ftppesca/40_1_95-103.pdf>. Acesso em: 18 nov. 2015.

Valente, B. S.; Xavier, E. G.; Pereira, H. S.; Pilotto, M. V. T. Compostagem de resíduos da filetagem de pescado marinho e casca de arroz. Revista Brasileira de Saúde e Produção

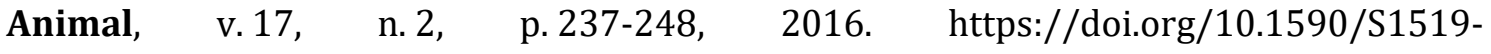
99402016000200011

Vieira, R. H. S. F. Microbiologia, higiene e qualidade do pescado: teoria e prática. São Paulo: Livraria Varela, 2004.

Xavier, A. Z. P.; Vieira, G. D. G.; Rodrigues, L. O. M.; Valverde, L. O.; Pereira, V. S. Condições higiênico-sanitárias das feiras-livres do Município de Governador Valadares. Governador Valadares: Universidade Vale do Rio Doce, 2009. (Trabalho de conclusão de curso).

Informação da Licença: Este é um artigo Open Access distribuído sob os termos da Licença Creative Commons Attribution, que permite uso irrestrito, distribuição e reprodução em qualquer meio, desde que a obra original seja devidamente citada. 